\title{
Interferometric temperature sensor based on a water- filled suspended-core fiber
}

\author{
Anahí Villalba (anahi@unizar.es), Juan Carlos Martín' (jcmartin@unizar.es) \\ Applied Physics Dept., Universidad de Zaragoza, C/ Pedro Cerbuna 12, 50009 Zaragoza (Spain) \\ †-Corresponding author
}

\begin{abstract}
The performance of water-filled suspended-core fibers as key components of interferometric temperature sensors has been analyzed. Use of water is motivated by simplicity of use and by its thermo-optic and thermal expansion coefficients, which provide a good balance between sensitivity and wide temperature range (limited by the free spectral range of the interferometer). Two suspended core fibers have been tested. With one of them, the sensitivity observed is slightly better than the ones of most interferometers based on liquid-filled photonic crystal fibers, with a linear temperature range of $25^{\circ} \mathrm{C}$. With regard to the other one, its most remarkable feature is its temperature range, not limited by any interferometric property but by water melting and boiling temperatures, as its quotient between free spectral range and sensitivity is higher than $100^{\circ} \mathrm{C}$.
\end{abstract}

Keywords - optical fiber sensor, interferometry, photonic crystal fiber, temperature, water.

\section{Introduction}

Optical fiber sensors have been thoroughly investigated due to their relevant advantages such as immunity to electromagnetic field, small size, reliability, remote sensing ability and multiplexing capability. In recent years, photonic crystal fibers (PCFs) interferometers have been demonstrated and used to measure physical parameters such as temperature [1], pressure [1-4], refractive index [4-5], etc. In particular, suspended-core fibers (SCFs) with several large air-holes around the core offer promising opportunities for sensing applications: temperature [6-8], pressure [8,9], strain [9], torsion [9], strain-temperature [10], or angle-temperature [10]. Focusing on temperature sensing, various configurations and techniques have been proposed and demonstrated such as long period fiber Bragg gratings [11] or tapered PCFs [12]. However, these devices always show a temperature sensitivity strongly limited by the low thermo-optic and thermal expansion coefficient of PCF components (silica and air). One possibility to enhance the device temperature dependence consists of including liquids in the arrangement. Different techniques have been demonstrated, such as splicing hollow core PCFs surrounded by a fluid [1], or infiltrating liquids, either filling first ring holes around the core [13], just two holes with different refractive index [14] or all holes [15]. In the last case, isopropanol is the most used filling liquid $[3,15]$ and the trend is using liquids with high thermo-optic and thermal expansion coefficients [16].

This way, high sensitivity values are achieved. But in an interferometric sensor, sensitivity is not an entirely positive feature: as it is well-known, interferometric measurements are limited by the free spectral range (FSR) of the interferometer, so that the unambiguous range of measurement of the sensed magnitude is given by the quotient between FSR and sensitivity. Therefore, a high sensitivity in an interferometric sensor entails a reduced measurement range. For this reason, our goal is not sensitivity but rather a good compromise between sensitivity, wide measurement range and simplicity. In order to achieve so, we seek a liquid whose thermal expansion and thermo-optic coefficients are neither especially high nor low. On the other hand, as the liquid must be introduced by capillarity into the PCF, we consider convenient to use an SCF, whose holes are much bigger than those of conventional PCFs, and to look for a liquid with low viscosity. In addition, as the SCF section must be spliced between two single mode fibers (SMFs), a non flammable liquid would be very suitable. Finally, the SCF performance is usually optimized for the third window of communications, so a low absorption coefficient of the liquid in this wavelength range is also desired. Water meets all these conditions, apart from other non-negligible features: low price and easy to deal with as it is not toxic. For all these reasons, it seems to us that trying an interferometer based on a water-filled SCF modal interferometer is worth it. 


\section{Experimental setup}

(a)

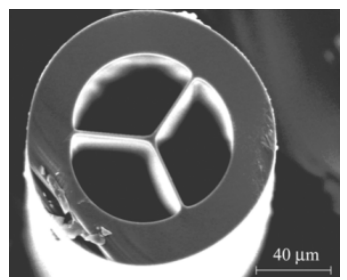

(b)

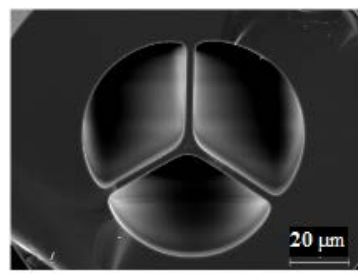

Fig. 1. Scanning electron microscope image of the cross section of the SCF 1 (a) and of the SCF 2 (b).

Two SCFs have been used in these experiments, fabricated by Laboratorio de fibras ópticas de la Universidad de Valencia. Cross section images of both can be seen in Fig. 1. SCFs 1 and 2 present cores with $4.5 \mu \mathrm{m}$ and $4.2 \mu \mathrm{m}$ and their outer diameters are $130 \mu \mathrm{m}$ and $73.7 \mu \mathrm{m}$, respectively. Before filling each SCF we cut it with a manual cleaver. Then, we introduced it into a syringe needle, sealed the joint, immersed it in water for some minutes and kept the plunger tight after having pulled it. Due to capillarity, the water was infiltrated gradually into the microholes of the SCF. Then, both ends of the water-filled SCF were spliced to standard SMFs, one of them finishing in an aluminum coating with a reflection coefficient of 0.73 .

Fig. 2 shows the experimental setup employed. The SLED light is led by the circulator to the water filled SCF contained in a thermostated bucket. Thanks to the mirror at the end of the fiber, light is reflected, so it passes again through the SCF and the circulator leads it to the OSA. This configuration is more suitable for remote sensing than the transmission setups usually proposed in the Literature: a remote probe based on a transmission setup would require a cable containing an input and an output fiber, with the sensitive component in its end, while the SCF together with the mirrored SMF can be placed at the end of a single fiber as long as necessary, providing a more compact solution.

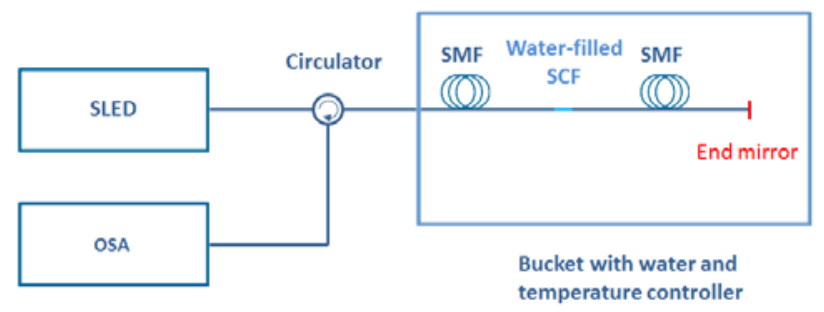

Fig. 2. Experimental setup used for temperature sensing measurements.

For the splicing procedure a commercial fusion splicer was used. For both SCFs we tested different kinds of splices in order to achieve the maximum transmission and a sharp interference figure. On the other hand, splicing involves at least a prefusion stage in which part of the water evaporates. For the sake of repeatability, several prefusions were carried out until $1 \mathrm{~mm}$ of the SCF fiber end was emptied (so, in the end $2 \mathrm{~mm}$ of SCF fiber were emptied: $1 \mathrm{~mm}$ for each fiber end). The width of the empty region can be easily controlled by means of the splicer microscope, as full and empty regions appear with clearly distinguishable grayscale tones.

\section{Results and discussion}

Figures 3 and 4 correspond to interferometers 1 and 2, built with $2.2 \mathrm{~cm}$ of SCF 1 and $2.0 \mathrm{~cm}$ of SCF 2, respectively. They show several transfer function spectra of the whole setup (output power at the OSA port divided by input power at the SLED port), measured at different temperatures. Spectra obtained are the result of two effects: interference of several modes excited in the SCF together with a certain birefringence, as the symmetry of the SCF cross section may become distorted close to the SMFSCF splices. A remarkable difference between Figs. 3 and 4 come from the number of interfering modes. Fig. 3 shows a typical bimodal interference pattern, while Fig. 4 shows an interference pattern due to more than two modes. On the other hand, the temperature shift trend observed in the transfer function is opposite in both cases: as temperature decreases, the transfer function spectrum redshifts for SCF 1 (Fig 3) and it blueshifts for SCF 2 (Fig 4). This shift trend is not surprising provided that SCF 1 and SCF 2 are totally different fibers. Therefore, their modes are different and so do their phase velocities' temperature dependence. Finally, the characteristic FSR of each interferometer can be appreciated: $16 \mathrm{~nm}$ for interferometer 1(Fig. 3) and $27 \mathrm{~nm}$ for interferometer 2 (Fig. 4). 


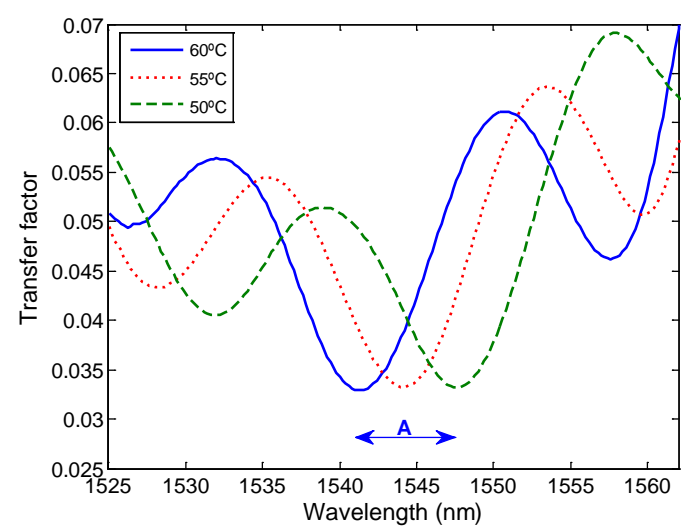

Fig. 3. Spectrum of the setup transfer function with $2.2 \mathrm{~cm}$ of SCF 1, for different temperatures.

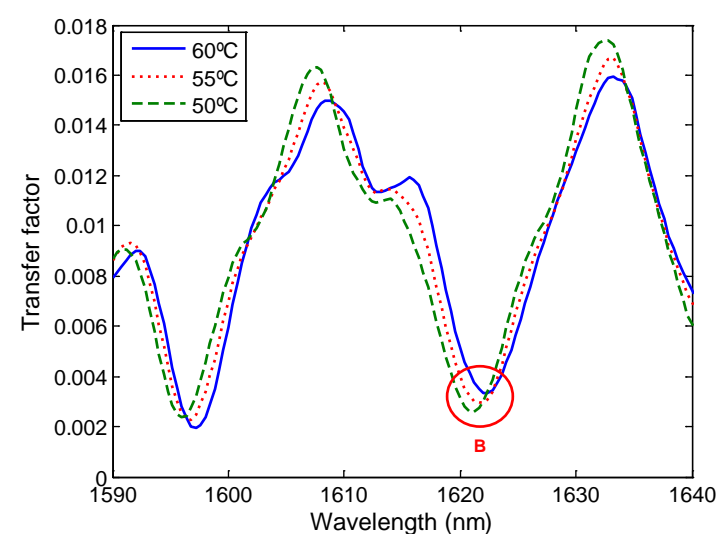

Fig. 4. Spectrum of the setup transfer function with $2.0 \mathrm{~cm}$ of SCF 2, for different temperatures.

Figures 5 and 6 show how the wavelengths of dips A and B evolve with temperature, respectively. Taking into account that the thermal expansion coefficient of water is not linear [17], there is no fundamental reason why the displacement of the dips with temperature should be linear. However, Figs. 5 and 6 show experimental series which admit a good linear fit, obviously an advantageous feature for a sensor.

With regard to interferometer 1 , the slope of the fitting straight obtained corresponds to a temperature sensitivity of $-541 \mathrm{pm} /{ }^{\circ} \mathrm{C}$. This sensitivity is higher than the ones achieved with other PCFs homogeneously filled with isopropanol or other liquids $[3,15,16]$, perhaps because with SCFs the overlap between liquid and modes is higher than for other PCFs, which compensates the lower thermo-optic and expansion coefficients of water with regard to other liquids (more sensitive interferometers based on liquid-filled PCFs have been reported [12,14,15], but their manufacture requires selective filling of just some holes of the PCF, that is to say, a more complex construction procedure). The quotient between FSR and sensitivity yields a range of $25^{\circ} \mathrm{C}$. Then, temperature can be measured beyond doubt along a range that broad. Notice that the range shown in Fig. 5 is much broader, so linearity in a range of $25^{\circ} \mathrm{C}$ is guaranteed.

For the interferometer 2 , the temperature sensitivity obtained is $165 \mathrm{pm} /{ }^{\circ} \mathrm{C}$. In this case, the quotient between FSR and sensitivity results in a temperature range beyond the limit given by melting and boiling temperatures, so we can determine a huge range of temperatures beyond doubt. 


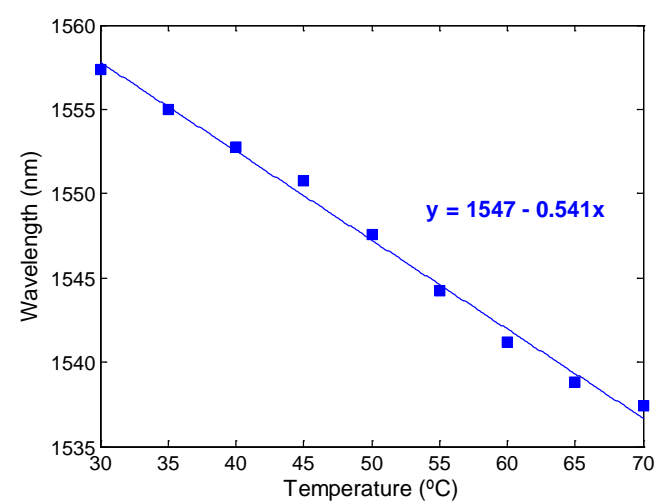

Fig. 5. Wavelength corresponding to the minimum at dip A, as a function of temperature (squares), and linear fit.

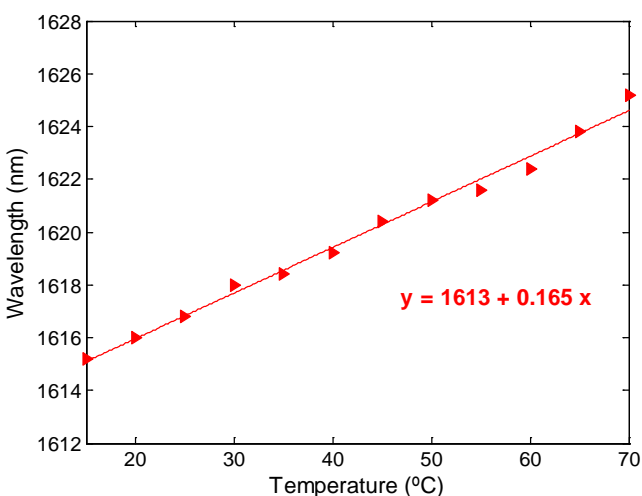

Fig. 6. Wavelength corresponding to the minimum at dip B, as a function of temperature (triangles), and linear fit.

\section{Conclusions}

In conclusion, possibilities of a water-filled SCF as key component of a temperature sensor based on a modal interferometer have been demonstrated. Compared with other liquids previously proposed for this application, water facilitates strongly the manufacturing process as, apart from its matchless availability, it is neither toxic nor flammable, which simplifies the splicing procedure. In addition, its low viscosity together with the size of the SCF holes favors a fast infiltration. With regard to the results obtained, they exhibit a good compromise between two desirable but opposite features: sensitivity and sensor range. A proper choice of the SCF enforces one feature or the other, so that the choice of a particular SCF would depend on the specific temperature sensing application. It has to be emphasized that in the case of SCF 1 sensitivities obtained are slightly better than the ones of other interferometers based on PCFs homogeneously filled with other liquids with higher thermo-optic and thermal expansion coefficients. On the other hand, in the case of SCF 2 it is remarkable that the quotient between FSR and sensitivity is so high that the sensor range is limited by water melting and boiling temperatures rather than by its interferometric properties.

\section{Acknowledgement}

The authors thank Antonio Díaz for his help. This work was supported in part by the national program MINECO TEC 201346643-C2-2-R and in part by the Gobierno de Aragón-Fondo Social Europeo under Grant TOL-T76.

\section{References}

[1] B. Dong, E. J. Hao, Core-offset hollow core photonic bandgap fiber-based intermodal interferometer for strain a temperature measurements, Appl. Optics, 50 (2011) 3011-3014.

[2] G. Coviello, V. Finazzi, J. Villatoro, V. Pruneri, Thermally stabilized PCF-based sensor for temperature measurements up to $1000^{\circ} \mathrm{C}$, Opt. Express, 17 (2009), 21551-21559.

[3] S.-j. Qiu, Y. Chen, F. Xu, Y.-q. Lu, Temperature sensor based on an isopropanol-sealed photonic crystal fiber in-line interferometer with enhanced refractive index sensing, Opt. Lett., 37 (2012) 863-865.

[4] K. Mileńko, D. J. J. Hu, P. P. Shum, T. Zhang, J. L. Lim,Y. WangT. R. Woliński, H. Wei, W. Tong, Photonic crystal fiber tip interferometer for refractive index sensing, Opt. Lett., 37 (2012) 1373-1375. 
[5] S.-j. Qiu, Y. Chen, J.-l. Kou, F. Xu, Y.-q. Lu, Miniature tapered photonic crystal fiber interferometer with enhanced sensitivity by acid microdroplets etching, Appl. Optics, 50 (2011) 4328-4332.

[6] Orlando Frazão, S .F. O. Oliveira, J. Viegas, José M. Baptista, José L. Santos, Jens Kobeller, Kay Shcuster, All fiber Mach-Zehnder interferometer based on suspended twin-core fiber, IEEE Phot. Tech. Lett., 22 (2010) 1300-1302.

[7] Susana F. Oliveira Silva, José L. Santos, Jens Kobelke, Kay Schuster, Orlando Frazão, Simultaneuos measurement of three parameters using an all-fiber Mach-Zehnder interferometer based on suspended twin-core fibers, Opt. Eng., 50 (2011) 030501-1-030501-3.

[8] S.H. Aref, M.I. Zibaii, M. Kheire, H. Porbeyram, H. Latifi, F.M. Araújo, L.A. Ferreira, J.L. Santos, J. Kobelke, K. Schuster, O. Frazão, Pressure and temperature characterization of two interferometric configurations based on suspended-core fibers, Opt. Comm., 285 (2012) 269- 273.

[9] Zhengyong Lu, Chuang Wu, Ming-Leung Vincent Tse, Hwa-Yaw Tam, Fabrication, characterization, and sensing applications of a high-birefringence suspended-core fiber, J. Lightwave Technol., 32 (2014) 2113-2122.

[10] Sergio Rota-Rodrigo, Manuel López-Amo, Jens Kobelke, Kay Schuster, José Luis Santos, Orlando Frazão, Multimodal interferometer based on a suspended core fiber for simultaneous measurement of physical parameters, J. Lightwave Technol., 33 (2015) 2468-2473.

[11]Z. Sun, Y.-g. Liu, Z. Wang, B. Tai, T. Han, B. Liu, W. Cui, H. Wei, W. Tong, Long period grating assistant photonic crystal fiber modal interferometer, Opt. Express, 19 (2011) 12913-12918.

[12]Yang Xue, Yong-Sen Yu, Rui Yang, Chuang Wang, Chao Chen, Jin-Chun Guo, Xuan-Yu Zhang, Cong-Cong Zhu, Hong-Bo Sun, Ultrasensitive temperature sensor based on an isopropanol-sealed optical microfiber taper, Opt. Lett., 38 (2013) pp. 1209-1211.

[13]Y. Peng, J. Hou, Y. Zhang, Z. Huang, R. Xiao, Q. Lu, Temperature sensing using the bandgap-like effect in a selectively liquid-filled photonic crystal fiber, Opt. Lett., 38 (2013) 263-265.

[14]S. Wang, Y-g. Liu, Z. Wang, T. Han, W. Xu, Y. Wang, S. Wang, Intermodal interferometer based on a fluid-filled two-mode photnoic crystal fiber for sensing applications , Appl. Optics, 52 (2013) 3166-3171.

[15]H. Liang, W. Zhang, P. Geng, Y. Liu, Z. Wang, J. Guo, S. Gao, S. Yan, Simultaneous measurements of temperature and force with high sensitivities based on filling different index liquids into photonic crystal fiber, Opt. Lett., 38 (2013) 1071-1073.

[16]R. Jha, J. Villatoro, G. Badenes, Ultrastable in reflection photonic crystal fiber modal interferometer for accurate refractive index, Appl. Phys. Lett., 93 (2008) 191106.

[17]Loren G. Helper, Thermal expansion and structure in water and aqueous solutions, Can. J. Chem., 47 (1969) 46134617. 


\section{Figure Captions}

Fig. 1. Scanning electron microscope image of the cross section of the SCF 1 (a) and of the SCF 2 (b).

Fig. 2. Experimental setup used for temperature sensing measurements.

Fig. 3. Spectrum of the setup transfer function with $2.2 \mathrm{~cm}$ of SCF 1, for different temperatures.

Fig. 4. Spectrum of the setup transfer function with $2.0 \mathrm{~cm}$ of SCF 2, for different temperatures.

Fig. 5. Wavelength corresponding to the minimum at dip A, as a function of temperature (squares), and linear fit.

Fig. 6. Wavelength corresponding to the minimum at dip B, as a function of temperature (triangles), and linear fit. 